\title{
IN MEMORIAM RUDOLF BULTMANN
}

\author{
* 20. 8. I $884 \dagger$ 30. $7 \cdot 1976$
}

Abschied nehmen von einem großen Gelehrten heißt nicht selten Rückschau halten auf ein Lebenswerk, dem eine gewandelte, vorwärts drängende Gegenwart in Wahrheit nur noch einen zwar ehrenvollen, aber inzwischen längst überholten Platz in der Vergangenheit zuerkennen kann. Von Rudolf Bultmann, der am 30. Juli 1976 im hohen Alter von fast 92 Jahren von uns gegangen ist, läßt sich so nicht sprechen. Dafür ist die Wirkung, die von ihm ausgegangen ist und bis heute ausgeht, zu stark und mächtig - weit über den Bereich seines eigensten wissenschaftlichen Arbeitsfeldes und die Grenzen einer Sprache, einer Kirche und einer Konfession hinaus in der ganzen Welt. Unwillkürlich werden viele dabei sofort an die leidenschaftliche Erregung denken, die das mit seinem Namen verbundene Schlagwort einer Entmythologisierung der neutestamentlichen Botschaft seit Anfang der Vierzigerjahre ausgelöst hat. Wie unterschiedlich und gegensätzlich die Reaktion darauf war und bis heute geblieben ist - bei den einen Dank und Zustimmung, bei den anderen Ablehnung bis zur Verketzerung - ist zur Genüge bekannt. Doch soll davon an dieser Stelle nicht sofort die Rede sein, sondern von dem auch von seinen wissenschaftlichen Gegnern nicht bestrittenen Rang und der umfassenden Weite seiner Arbeit, die jeden Fachgenossen nötigen, in der Forschung sich nicht nur mit ihm, sondern die eigene Arbeit an der seinen zu messen; seinem beharrlichen Ruf zur Sache, der Kraft seiner Einsichten und der Strenge seiner Kritik, die nach Gründen fragt und sich mit gängigen Traditionen und Konventionen nicht zufrieden gibt. Kein Zweifel, dass kritische Gespräch mit ihm kann und darf nicht abbrechen, wenn anders die Arbeit unserer Wissenschaft nicht verhängnisvoll absinken soll.

Bultmann verdankt sein hohes Ansehen der wissenschaftlichen Leistung seines biographisch einfach und geradlinig verlaufenen Gelehrtenlebens. Der oldenburgische Pfarrerssohn studierte in Tübingen, Berlin und Marburg in der Schule vor allem einiger bedeutender Vertreter der historischkritischen Theologie, Adolf von Harnack, Adolf Jülicher, Johannes Weiß und des systematischen Theologen Wilhelm Herrmann, wurde igio aufgrund einer von J. Weiß angeregten Dissertation Der Stil der paulinischen Predigt und die kynisch-stoische Diatribe in Marburg promoviert und 1912 für Neues Testament habilitiert, I 916 als Extraordinarius nach Breslau und 1920 als Nachfolger W. Boussets nach Gießen berufen und kehrte I92 I nach Marburg zurück, das fortan auch über seine Emeritierung (195I) hinaus seine Wirkungsstätte blieb. Seine in diesen Jahrzehnten stetig gewachsene 
Arbeit sollte mehr und mehr die Bereiche seiner Wissenschaft in einer erstaunlichen, von kaum einem anderen in gleicher Weise erreichten und überschauten Weitse umspannen, aber zugleich auch, wie viele seiner Veröffentlichungen zeigen, tief in das Gebiet der klassischen Altertumswissenschaften, der hellenistisch-orientalischen Religionsgeschichte und der älteren und neueren Philosophie vorstoßen.

Im Unterschied zu den ihm befreundeten, früh verstorbenen Kollegen J. Weiß, W. Bousset und W. Heitmüller - auch der frühere bedeutende Inhaber seines Breslauer Lehrstuhles W. Wrede gehört in ihre Reihe - war es Bultmann vergönnt, seinen Weg zu Ende zu gehen und, soweit das überhaupt von einem Menschen gesagt werden kann, sein Lebenswerk abzuschließen. Gewiß nahmen seine Kräfte in den letzten Jahren spürbar ab, so da $\beta$ er den Fortgang der theologischen Arbeit nur noch fragmentarisch, wenngleich stets mit der ihm eigenen kritischen Aufmerksamkeit verfolgen konnte. Doch geben die zahlreichen Veröffentlichungen des Hochbetagten aus den letzten Jahrzehnten einen zur Genüge deutlichen Eindruck davon, wie intensiv er auch mit schwindender Kraft seine gewohnte Arbeit weiterführte und ihre Wirkung unvermindert anhielt. Einige davon haben in dem von E. Dinkler eingeleiteten und herausgegebenen Sammelband seiner Exegetica (1967) Aufnahme gefunden; andere sind in der angefügten, umfangreichen Bibliographie aufgezählt. Nicht zu vergessen auch seine erst jüngst erschienenen Kommentare zu den drei fohannesbriefen ( 1967 ) und die ebenfalls von E. Dinkler eingeleiteten und herausgegebenen, wenigstens teilweise von Bultmann ausgearbeiteten Bruchstücke seiner letzten Vorlesung über den von ihm besonders geliebten Zweiten Korintherbrief (1976) bis hin zu seiner wohl letzten exegetischen Studie Die Interpretation von $M k$ 4, 3-9 seit Fülicher (Festschrift W. G. Kümmel, 1975, S. 30-34). Auch wer wie ich selbst die Freude hatte, ihn bis in die Jahre und Monate vor seinem Tod besuchen zu dürfen, erlebte ihn im doppelten Sinn des Wortes ganz als den Alten, in der Präsenz seines Geistes, der Klarheit seines Urteils und nicht zuletzt in seiner gelassenen Heiterkeit und Güte freundschaftlicher Zuwendung.

Von kaum einer der Arbeiten Bultmanns läßt sich ernstlich behaupten, daß sie überholt sei, auch wenn Erkenntnis und Diskussion im einzelnen weitergegangen sind. Nicht wenige sind zu Marksteinen und Standardwerken der neutestamentlichen Forschung geworden. Das gilt schon für sein erstes noch in seiner Breslauer Zeit entstandenes Werk Die Geschichte der synoptischen Tradition (192 I), das zusammen mit der etwa zur gleichen Zeit unabhängig von Bultmann konzipierten Programmschrift von M. Dibelius 'Die Formgeschichte des Evangeliums' (I919) die fortan nach Dibelius' Titel benannte formgeschichtliche Erforschung der Synoptischen Evangelien begründete und für sie bis heute maßgebend blieb. Sie war in vielfacher Hinsicht vorbereitet und lag sozusagen 'in der Luft'.' Die bis dahin vorherrschende

${ }^{1}$ Vgl. zum folgenden Ph. Vielhauer, Geschichte der urchristlichen Literatur (1975), S. $278 \mathrm{ff}$. 
Literar- und Quellenkritik war an ihr vorläufiges Ende gekommen und hatte auch nach Bultmann und Dibelius ihre Feuerprobe bestanden. Ihre Ergebnisse wurden auch von der Formgeschichte nicht angefochten (MarkusPriorität; Zwei-Quellen-Theorie). Aber auch die Kritik an der älteren historisch-kritischen Forschung in der Leben-Jesu-Aera hatte bereits kräftig eingesetzt. Wrede hatte in seinem 'Messiasgeheimnis in den Evangelien' I 90 I die traditionelle Annahme, daß sich aus dem Markusevangelium der Ablauf der Wirksamkeit des historischen Jesus und sogar seine innere Entwicklung noch mit einiger Sicherheit rekonstruieren ließen, als Irrtum erwiesen. Wellhausen kam in seinen kurzen Kommentaren zu den drei ersten Evangelien und der als Vorwort $z u$ ihnen gedachten Einleitung (sämtlich in den ersten Jahren dieses Jahrhunderts erschienen) zu dem gleichen Ergebnis, aber wurde insbesondere dadurch zu einem Schrittmacher der späteren Formgeschichte, da $\beta$ er ausgehend von der schon von Herder mit Recht betonten Erkenntnis, daß die letzte Quelle der Evangelien die mündliche Überlieferung ist, diese als zerstreutes, ursprünglich in relativ kleinen Einheiten einzeln umlaufendes Gut charakterisierte, das erst nachträglich von den Verfassern der Evangelien zu einem Ganzen verbunden wŭrde. Damit war die von K. L. Schmidt in seinem Buch Der Rahmen der Geschichte Fesu (I919) systematisch durchgeführte erste Aufgabe der formgeschichtlichen Forschung klar bezeichnet, die Scheidung von Tradition und Redaktion. Das bleibende Verdienst der beiden eigentlichen Meister der Formgeschichte besteht jedoch darin, daß sie über diesen ersten Schritt hinaus in konsequenter Anwendung der vor allem durch H. Gunkel am Alten Testament erprobten und bewährten formgeschichtlichen Methode auf die Evangelientexte neue Wege zeigten, die Formen und Gesetze der vorliterarischen, mündlichen Jesus-Überlieferung sowie ihre Verankerung im Glauben und Leben der Gemeinde ('Sitz im Leben') zu erkennen und auf diese Weise Geschichte und Wesen des Urchristentums zu erhellen. Daß beide in ihrer Arbeit verschieden vorgingen, Bultmann auf dem Wege einer Analyse der Einzelstücke, Dibelius ausgehend von einer 'Konstruktion der Anschauungen und Bedürfnisse der Gemeinde' (Vielhauer), daß sie sich auch in der Terminologie und manchen Einzelheiten unterscheiden, kann hier außer Betracht bleiben. Aufs Ganze gesehen ist die Übereinstimmung ihrer Ergebnisse erstaunlich, ergänzten und korrigierten sie sich aufs beste und erwies sich der von ihnen gewiesene Weg als höchst fruchtbar.

$\mathrm{Da} \beta$ die in der heutigen neutestamentlichen Wissenschaft geradezu als klassisch geltenden Werke der Formgeschichte sofort eine Wende heraufgeführt hätten, läßt sich freilich keineswegs behaupten. Wie sich schon an den Erscheinungsjahren ihrer erst seit den Fünfzigerjahren rapide angewachsenen Neuauflagen und Übersetzungen ablesen läßt, brauchten sie eine beträchtliche Zeit sich durchzusetzen. Sehe ich recht, nahm im wesentlichen nur die internationale Fachwelt und auch diese nur partiell von ihnen Kenntnis 
und zwar überwiegend mit Kritik, natürlich vor allem die konservative, aber nicht minder auch die historisch-kritische Bibelwissenschaft, und gab ihnen allenfalls mit distanziertem Respekt einen Außenseiterplatz. Höchstens, daß man sie pflichtschuldigst notierte und einige ihrer Ergebnisse zu einer rein formalen Klassifikation der Evangelientexte benutzte. Andere lehnten sie mit erregtem Protest ab und warnten vor der neuen Schulrichtung. Es wäre interessant, den Gründen und Ursachen für diese zunächst relativ geringe Wirkung der Formgeschichte nachzugehen. Zweifellos haben dabei die turbulenten Zeitumstände nach dem ersten Weltkrieg, später der zweite und dazwischen die Drosselung des wissenschaftlichen Austausches in und zwischen den Ländern durch das Gewaltregime in Deutschland eine Rolle gespielt, auch die erst in den letzten Jahrzehnten glücklicherweise abgebauten Mauern zwischen den Kirchen und Konfessionen in aller Welt und ihrer Theologie. Einer der Hauptgründe dürfte jedoch in eben dieser Theologie selbst zu suchen sein, die noch im Banne eines gewissen historischen Positivismus besonders durch Bultmanns Aufweis der starken Einwirkung der Gemeindetheologie auf die Evangelienüberlieferung ihre eigenen Fundamente bedroht sah-nach Meinung der einen die 'objektiven Heilstatsachen', nach Meinung der anderen der 'historische Jesus'. Tatsache ist jedenfalls, daß vor allem Bultmann fortan in der Theologie und bald auch in der Laienwelt weithin als Vertreter einer übersteigerten historischen Skepsis oder gar als gefährlicher Destrukteur der biblischen Úberlieferung galt.

Das aus der formgeschichtlichen Arbeit am Neuen Testament erwachsende, weiterer Klärung bedürftige exegetisch-theologische Grundproblem kommt in Bultmanns sprödem ersten großen Werk noch nicht ausdrücklich zur Sprache. Es steht aber sozusagen zwischen den Zeilen, und Bultmann selbst hat es bald nach Erscheinen seiner 'Geschichte der synoptischen Tradition' seit Beginn der Zwanzigerjahre in zahlreichen Veröffentlichungen unter mannigfaltigen Aspekten zur Sprache gebracht. Es ist damit gegeben und aufgegeben, daß die Interpretation des Neuen Testaments es nicht mit einer bloßen Summe von konstatierbaren oder rekonstruierbaren Fakten und Vorgängen der Vergangenheit zu tun hat, sondern mit einer im Glauben verstandenen und sehr verschiedenartig gedeuteten Geschichte, und zwar gedeutet in Sprache, Vorstellungs- und Denkweise einer fremden, vergangenen Zeit und Welt. Für eine Exegese, die dem Anspruch der Texte genügen will, ergibt sich daraus nach Bultmann die Aufgabe, die in ihnen bekundete Geschichte nicht nur wie Objekte der Natur zu beschreiben und einzuordnen, sondern sie in einer gewandelten Welt zu verstehen und verständlich zu machen und damit ihren Anruf dem Menschen heute vernehmbar werden zu lassen. Denn ein Verstehen der Geschichte - aller Geschichte und so auch der im Neuen Testament bekundeten-gibt es einzig und allein darum, weil der ihr begegnende Mensch selbst ein Stück Geschichte ist, bewegt von denselben Fragen seiner Existenz wie die Menschen 
einer früheren Zeit, auch wenn er sie im einzelnen sehr anders ausspricht und beantwortet. Diese Fragen, ob ausgesprochen oder unausgesprochen, wollen in die Begegnung mit der Geschichte eingebracht werden, denn nur so und nicht anders kommt es zu einem Hören dessen, was sie über den Abstand der Zeiten hinweg sagen wollen.

Die Bedeutung dieser Gedanken eines dialogischen Geschichtsverständnis für eine verantwortliche Predigt der Kirche, um die Bultmann bis an sein Lebensende besorgt war, ist offenkundig. Er selbst hat sie fortan als Forscher, Lehrer und Prediger beharrlich zur Geltung gebracht. In ihren Grundzügen schon auf den ersten Seiten seines allgemeinverständlichen Fesusbuches (1926) bewundernswert klar und einfach ausgesprochen, kündigen sie bereits die großen hermeneutischen und theologischen Themen seines weiteren Lebenswerkes an: Glauben und Verstehen, Geschichte und Offenbarung. Man sollte darum sein vielfach aufgelegtes, über die ganze Welt verbreitetes Büchlein lesen und wieder lesen nicht nur mit der Befriedigung, daß seine kritische Analyse der Tradition also doch nicht in ein völliges Vakuum geführt und in dem angeblichen Nebel einer 'anonymen Gemeindetheologie' noch einiges Wesentliche aus Jesu Geschichte und Verkündigung stehen gelassen habe. Vielmehr unter dem Aspekt der in der Einleitung - sehr bezeichnend überschrieben 'Die Art der Betrachtung'- entwickelten Gedanken, die ihn befähigten, insbesondere den inneren Zusammenhang der prophetisch-eschatologischen Predigt Jesu und seiner radikalen Verkündigung des Willens Gottes eindrucksvoll zu profilieren. Anmerkungsweise sei hier schon erwähnt, daß entgegen einer verbreiteten Meinung, wie er mir selbst noch bei meinem letzten Besuch im Frühjahr 1976 ausdrücklich bestätigte, nicht sein Jesusbuch unter dem Einfluß der Philosophie M. Heideggers entstanden ist, sondern umgekehrt dieser, soeben nach Marburg berufen, aufgrund der Lektüre des Buches die Verbindung mit Bultmann suchte, woraus sich dann allerdings eine unvergleichliche Zusammenarbeit im Geben und Nehmen entwickelte, die wie vielen anderen in jenen Marburger Jahren so auch mir unvergeßlich bleiben wird. Kaum nötig zu sagen, daß es mir bei dem eben Gesagten nicht darum geht, die 'Originalität' bestimmter wichtiger Gedanken der Theologie Bultmanns zu verteidigen. Wohl aber liegt mir daran, die irrige Vorstellung einer späteren Überfremdung seines Denkens abzuwehren, das in Wahrheit konsequent aus seiner exegetischen Arbeit erwuchs und das er auch weiterhin selbständig entwickelte.

Ohne die hier nur skizzierten Grundgedanken ist auch Bultmanns sonstige Arbeit am Neuen Testament nicht zu denken. Sie durchziehen ebenso seine zahlreichen Studien zur paulinischen und johanneischen Theologie, die ersteren zusammengefaßt in seiner Theologie des Neuen Testaments ( $\left.{ }^{6} \mathrm{I} 965\right)$, die letzteren in seinem durch mehrere Spezialuntersuchungen vorbereiteten monumentalen Kommentar zum Fohannesevangelium, dem 1967 noch der zu den drei Fohannesbriefen folgte. $\mathrm{Zu}$ nennen sind aber auch seine zahlreichen Artikel 
schon in RGG (2. Aufl.), und vollends im Theologischen Wörterbuch zum Neuen Testament. Schließlich neben diesen neutestamentlichen Werken die auch für die systematische Theologie und teilweise für die Philosophie wichtig gewordenen Abhandlungen in seinen unter dem programmatischen Titel Glauben und Verstehen gesammelten vier Aufsatzbänden (I ${ }^{6}$ I 966 ; II ${ }^{5}{ }_{1968 ;}$ III ${ }^{3}{ }_{1965} ;$ iv ${ }^{2}{ }_{1967}$ ) sowie sein allgemein verständliches, mehrfach aufgelegtes und übersetztes Buch Das Urchristentum im Rahmen der antiken Religionen (zuletzt: rororo 1962 ), in dem außer dem Urchristentum selbst Altes Testament und Judentum, Stoa und Gnosis eine knappe, meisterhaft durchsichtige Darstellung gefunden haben. Besonders erwähnt sei dabei nochmals die von Anfang an große Bedeutung, die die Antike, vornehmlich die griechische, in Bultmanns gesamten Werk zukommt und ihm die Ehrendoktorwürde der Philosophischen Fakultät eintrug. Viele Titel seiner umfänglichen Bibliographie (Exegetica S. 483-507) geben davon Zeugnis. Neben Aufsätzen über Christentum und Antike, das griechische und christliche Verständnis der Freiheit, der Geschichte u. a. möchte ich besonders auf Bultmanns Beitrag Polis und Hades in der Antigone des Sophokles in der Festschrift für K. Barth (1935) hinweisen, der erfrenlichenweise in Glaube und Verstehen Bd. II S. 20-3I wieder abgedruckt ist, weil er mit seiner Konfrontation von Kreon und Antigone damals im Kampf der Bekennenden Kirche gegen das NS-Regime für jeden Aufmerksamen ein unmißverständlicher Ruf zur Besinnung war und darum auch als geschichtliches Dokument jener Zeit nicht in Vergessenheit geraten sollte. Aber auch abgesehen von diesem aus aktuellem Anlaß verfaßten Aufsatz war für Bultmann die Beschäftigung mit der außerchristlichen Antike niemals eine aus antiquarischem Interesse geborene gelehrte Liebhaberei, sondern diente ihm dazu, durch Herausarbeitung des Gemeinsamen sowohl wie der Unterschiede das Besondere der urchristlichen Botschaft zu verstehen. Freilich war die Lektüre der Griechen für ihn auch eine unversiegbare Quelle der Erholung und Freude. Er pflegte sie darum über lange Jahrzehnte im kleinen Kreis befreundeter Kollegen aus verschiedenen Fakultäten in seiner Graeca. Ich selbst erinnere mich dieser Abende, an denen in meiner Marburger Zeit auch H. G. Gadamer, G. Krüger und H. Schlier teilnahmen, mit besonderer Freude.

Bultmanns erstaunlich weit ausgreifendes, vielseitiges Lebenswerk war und blieb jedoch von dem fundamentalen Anliegen und Thema bestimmt, das seine historisch-kritische Arbeit und seine Theologie zu einer unauflöslichen Einheit werden ließ: das Verständnis der Geschichte nicht als einer bloßen Rekonstruktion gegebener objektiver Tatsachen, sondern im Sinne ihrer Vergegenwärtigung und ineins damit einer Klärung der grundsätzlichen Fragen und Voraussetzungen ihres Verstehens. Beidem zugleich galt Bultmanns Arbeit mit unbeirrter, gesammelter Energie. Sein theologischer Weg führte ihn darum zum Befremden mancher seiner früheren liberalen Lehrer, die darin nur eine jähe Flucht aus seiner angeblich übertriebenen 
historischen Skepsis zu erblicken vermochten, in die durch K. Barths 'Römerbrief' hervorgerufene stürmische Aufbruchsbewegung der sogenannten dialektischen Theologie, weil in ihr der christliche Glaube nicht mehr nur als ein allgemein religiöses oder kulturelles Phänomen verstanden wurde, sondern als Antwort auf das Wort des transzendenten Gottes, der dem Menschen in diesem Wort richtend und rettend begegnet. Ebenso führte ihn dieser Weg bald danach in die aktive Gliedschaft und in den Kampf der Bekennenden Kirche für eine unverfälschte Verkündigung gegenüber dem Ungeist des Nationalsozialismus. Die theologischen Wege Barths und Bultmanns haben sich freilich seitdem in vieler Hinsicht geschieden. Die für Bultmann entscheidende Erkenntnis, daß die im Neuen Testament bezeugte Geschichte sich dem Menschen heute nicht unmittelbar erschließt, ihm sozusagen nicht in den Schoß fällt und er, gebunden an die Verstehensmöglichkeiten seiner Zeit und Welt, ihren fordernden und begnadenden Anruf nicht zu hören vermag, war für Barth kein zentrales theologisches Problem. Bultmann dagegen sah es als eine vordringliche Aufgabe der Theologie an, jene Verstehensmöglichkeiten der Gegenwart zu reflektieren und die zeitbedingten Mauern zwischen dem in einer vergangenen Geschichte ergangenen Wort und dem Fragenden und Hörenden heute abzubauen, um ihn in seinem Unvermögen so erst recht auf das eigentliche und wahre Skandalon der Christusbotschaft zu weisen. So blieben diese Fragen, wie ihr freundschaftlicher und gerade darum rückhaltloser Briefwechsel zeigt, zwischen Barth und Bultmann zeitlebens kontrovers (vgl. K. Barth-R. Bultmann, Briefwechsel 1922-1966, insbes. S. $169 \mathrm{ff}$.).

Um so fruchtbarer, nach Meinung nicht weniger Theologen freilich verhängnisvoller, wurde für Bultmann die Begegnung mit der Existenzialphilosophie M. Heideggers, weil sie ihm dazu verhalf, sein zentrales theologisches Anliegen, erwachsen wie gezeigt aus seiner historisch-kritischen Arbeit und durch Barths Römerbrief (2. Aufl.) in eine neue Richtung gebracht, begrifflich zu klären und zu entfalten - nicht in der Richtung, wie viele ihm vorwerfen, die Theologie in Anthropologie aufzulösen, wohl aber beide in ihrer steten Wechselbeziehung zu bedenken, ohne dabei Gott zu einer bloßen Chiffre für menschliche Existenz werden zu lassen.

Wie bekannt verdichtete sich die Problematik in Bultmanns Theologie wie in einem Brennpunkt in dem über Jahrzehnte leidenschaftlich umstrittenen, 194 I gehaltenen Vortrag 'Neues Testament und Mythologie' (zuerst veröffentlicht in: Offenbarung und Heilsgeschehen, 194I). Welche Lawine er damit lostrat, konnte er damals ebensowenig wissen wie seine Zuhörer, denn ähnliche Gedanken hatte er längst zuvor schon vielfach ausgesprochen und veröffentlicht. Und es bleibt ein Widersinn, daß ausgerechnet Name und Werk dieses allen bloßen Modeerscheinungen und Schlagworten unzugänglichen Forschers und Theologen seitdem mit dem lauten Streit um oft nur halbverstandene Parolen verknüpft sind. 
Es ist hier nicht der Ort, auf den bekannten Inhalt der Schrift Bultmanns näher einzugehen. Überdies haben sich die erregten Wogen des Streites erfreulicherweise merklich gelegt, und die besonnene Arbeit zumal katholischer Theologen hat dazu beigetragen, Bultmanns Anstöße aufzunchmen und weiterzudenken. Mancher in der gelehrten Zunft unseres Faches wird sich vielleicht in den von Bultmann erneut und radikal gestellten theologischen Fragen für inkompetent oder an ihnen nicht interessiert erklären. Doch würde die Wissenschaft des Neuen Testamentes von Bultmann nicht nur Abschied nehmen, sondern ihm den Abschied geben, wenn sie sich beruhigt wieder auf das sturmfreie Feld bloßer Tatsachenforschung zurückzöge. Gerade der herausfordernde Anruf seiner Theologie darf nicht verstummen, und ebenso wenig darf die Bereitschaft aufhören, ihre Einsichten und Anstöße aufzunehmen und für die Interpretation des Neuen Testamentes fruchtbar zu machen. Die Unabgeschlossenheit und Offenheit jeglicher theologischer Arbeit, bedingt durch die jedem gesetzten Lebensgrenzen und erst recht durch die ihr aufgegebene Sache, hat gerade er mehr als einmal betont. Auch die, welche einst oder wieder im Zeichen der Leben-JesuTheologie aufgebrochen sind, können vielleicht eines Tages einsehen, daß die von Bultmann schon zu Beginn seines wissenschaftlichen Werkes erarbeitete und niemals preisgegebene Erkenntnis, daß das Kerygma der urchristlichen Tradition - das Evangelium den Evangelien - zeitlich und sachlich vorangeht, eine eminent positive und nicht destruktive Bedeutung hat. Ich könnte auch sagen: die, welche ausgezogen sind und ausziehen, eine Eselin zu suchen, haben die Chance, ein Königreich zu finden.

G. BORNKAMM 